\title{
Likert y Diferencial Semántico en la medición de actitudes hacia la química en estudiantes universitarios
}

Odetti, Héctor S. ${ }^{1}$ Tiburzi, María del C.; Mondino, Azucena M.; Güemes, René 0.

\section{Resumen}

Se presenta una comparación entre la Escala de Likert y el método del Diferencial Semántico con el objetivo de evaluar la actitud hacia la química y evidenciar si ambas técnicas pueden medir en forma equiparable dicha actitud en un grupo de alumnos ingresantes a la universidad.

El análisis de los resultados indica que ambas escalas detectan en forma general una actitud positiva de los alumnos frente a la química. Sin embargo, al analizar la concordancia entre ambas técnicas, se encuentra que ésta es casi nula.

${ }^{1}$ Cátedra de Química Inorgánica. Facultad de Bioquímica y Ciencias Biológicas. Universidad Nacional del Litoral. Santa Fe. Argentina. hodetti@fbcb.unl.edu.ar

${ }^{2}$ Los autores agradecen a la magíster Liliana Contini por el experto asesoramiento brindado en técnicas de análisis estadísticos. 